\title{
Socioeconomic status as a risk factor for dementia death: individual participant meta-analysis of 86508 men and women from the UK*
}

Tom C. Russ, Emmanuel Stamatakis, Mark Hamer, John M. Starr, Mika Kivimäki and G. David Batty

\section{Background}

Life-course socioeconomic factors may have a role in dementia aetiology but there is a current paucity of studies. Meta-analyses of individual participant data would considerably strengthen this evidence base.

\section{Aims}

To examine the association between socioeconomic status in early life and adulthood with later dementia death.

\section{Method}

Individual participant meta-analysis of 11 prospective cohort studies (1994-2004, $n=86508)$.

\section{Results}

Leaving full-time education at an earlier age was associated with an increased risk of dementia death in women (fully adjusted hazard ratio (HR) for age $\leqslant 14 \mathrm{v}$. age $\geqslant 16$ : $\mathrm{HR}=1.76,95 \% \mathrm{Cl} 1.23-2.53)$ but not men. Occupational social class was not statistically significantly associated with dementia death in men or women.

\section{Conclusions}

Lower educational attainment in women was associated with an increased risk of dementia-related death independently of common risk behaviours and comorbidities.

\section{Declaration of interest}

None.
Socioeconomic inequalities in cardiovascular disease ${ }^{1}$ and selected cancers $^{2}$ are well recognised. More recently, research attention has focused on such differentials in mental health, including depression $^{3}$ and other common mental disorders such as dementia. ${ }^{4-6}$ There is a suggestion that socioeconomic factors may have a role in the aetiology of dementia including lifetime manual occupation ${ }^{7}$ as well as various indicators of socioeconomic status in early life ${ }^{8}$ and lower educational attainment. ${ }^{8,9}$ However, because of the paucity of large-scale, well-characterised studies, the extant evidence is discordant and there has been inconsistent control for confounding variables. Thus, the precise nature of the socioeconomic status-dementia relationship remains unclear. Although individual studies undoubtedly have value in improving this evidence base, the pooling of raw data from multiple studies, which would also represent an important technical advance in this context, has yet to be utilised. We therefore undertook the first individual participant meta-analysis using data from 11 large, community-based cohort studies that held data on socioeconomic status, covariates and dementia death. The purpose of this paper is twofold: first, as a proof of principle that this methodology can be applied to the study of the role of socioeconomic position in the risk of dementia; and second, to add to the evidence base by further investigating the association between socioeconomic factors and dementia-related death.

\section{Method}

\section{Study samples}

Participants were taken from the Health Survey for England, ${ }^{10}$ a representative general population-based health examination study sampling individuals living in households in England. From 1994 to 2004,11 independent, cross-sectional studies with identical methodologies were conducted on an annual basis. Consenting study members $(89.6 \%)$ were followed up by linkage to the UK

*Preliminary results from this study were presented as a poster at the 2011 Alzheimer's Association International Conference in Paris.
National Health Service mortality registry. Study participants gave full informed consent and ethical approval was obtained from the London Research Ethics Council.

\section{Assessment of socioeconomic status}

During a household visit, interviewers collected information using computer-assisted personal interviewing modules. Information on occupational social class was collected during the interview and coded according to the Registrar General classification (professional (I), intermediate (II), skilled non-manual (IIINM), skilled manual (IIIM), part-skilled (IV) and unskilled (V)), a standard approach in the UK. ${ }^{11}$ Age on leaving full-time education was recorded as $<15,15,16,17,18,>18$, never went to school and still in full-time education. For this study occupational social class was coded into four groups: professional/intermediate (the referent), skilled non-manual, skilled manual and part-skilled/unskilled. Educational attainment was coded into three groups: 14 years or younger, 15 years old and 16 years or older (the referent).

\section{Assessment of other risk factors and comorbidities}

Smoking status (not a current smoker; $<5$ per day; $5-10$ per day; 10-15 per day; $15-20$ per day; $>20$ per day), weekly alcohol consumption (converted to units of alcohol), and history of cardiovascular disease and diabetes (including hyperglycaemia) were collected by self-report at interview. Individuals drinking above safe limits of alcohol were identified using gender-specific safe limits $(\leqslant 14$ units per week for women and $\leqslant 21$ units per week for men). ${ }^{12}$

Psychological distress was measured using the 12-item version of the General Health Questionnaire (GHQ-12), a widely used measure in population studies. ${ }^{13}$ A score of four is often used as a threshold to denote psychological distress, ${ }^{14}$ but since we have previously shown that even low levels of psychological distress that is, scores below four - are associated with an increased risk 
of dementia in these cohort studies, ${ }^{15}$ we adjusted for total GHQ-12 score as a continuous variable.

\section{Ascertainment of dementia}

Causes of death recorded on death certificates were coded using ICD-9 ${ }^{16}$ and ICD-10. ${ }^{17}$ Any mention of dementia death was identified using codes 290.0-290.4, 294.9, 331.0-331.2, and 331.9 for ICD-9, and F01, F03, F09, G30 and G31 for ICD-10.

\section{Statistical analyses}

We ascertained that the proportional hazards assumption had not been violated by inspecting the $\log (-\log ($ survival $))$ plot. We then used Cox proportional hazards models ${ }^{18}$ to compute study-specific hazard ratios with accompanying 95\% confidence intervals for the association between the measures of socioeconomic status and dementia death. Heterogeneity in the effect estimates between studies was examined using the $I^{2}$ statistic, which indicates the proportion of the total variation in the estimates that is due to between-studies variation. It varied between $0 \%$ and $36.9 \%$ depending on the measure of socioeconomic status used in the analysis. To obtain a conservative estimate, we pooled the study-specific effect estimates and their standard errors in random effects meta-analyses. Calendar time (days) was the time scale; for participants with no record of an event, the data were censored at the 15 February 2008.

Models were initially unadjusted, then a series of variables were added to the multivariable model: age, smoking status, alcohol consumption (units per week), baseline cardiovascular disease (yes/no), diabetes (yes/no), psychological distress (GHQ-12 score), occupational social class and educational attainment. Since, as described, the association between socioeconomic status and dementia has been reported to be different in men and women, ${ }^{19}$ gender-specific analyses were conducted. We compared the effects of controlling for different covariates/mediators on the magnitude of the association by examining a change in the size of hazard ratio rather than a change in significance level..$^{20}$

Individuals with data missing for one or more variables and those with no missing data were compared using Student's $t$-test for continuous variables and $\chi^{2}$ tests for categorical variables. The main analysis was based on participants with no missing data. In the sensitivity analysis, missing values for covariates were imputed with PASW statistics version 18.0 for Windows using five imputations based on maximum likelihood estimates. All other analyses were conducted using $\mathrm{R}$ version 2.15 .0 for Windows and the survival and metafor ${ }^{21}$ packages. The reporting of this study conforms to the STROBE statement. ${ }^{22}$

\section{Results}

The initial sample comprised 96605 individuals. The derivation of the sample is shown in Fig. 1. After removing individuals who declined linkage to mortality records $(n=10065)$ and for whom survival was incalculable or who had no cause of death recorded $(n=32)$ the maximum analytic sample comprised 86508 people (mean age 56.1 years, s.d. $=14.4$ ): 39125 men and 47383 women. Data were missing for occupational social class for 2325 individuals (analytic $n=84183$ ) and for educational attainment for 61 individuals (analytic $n=86447$ ). Table 1 shows the characteristics of study members from the 11 cohorts and pooled summaries.

Table 2 shows the baseline characteristics of the pooled sample according to occupational social class separately in men and women. Individuals from a lower occupational social class were older, were more likely to smoke, and had a somewhat greater likelihood of baseline cardiovascular disease, diabetes and

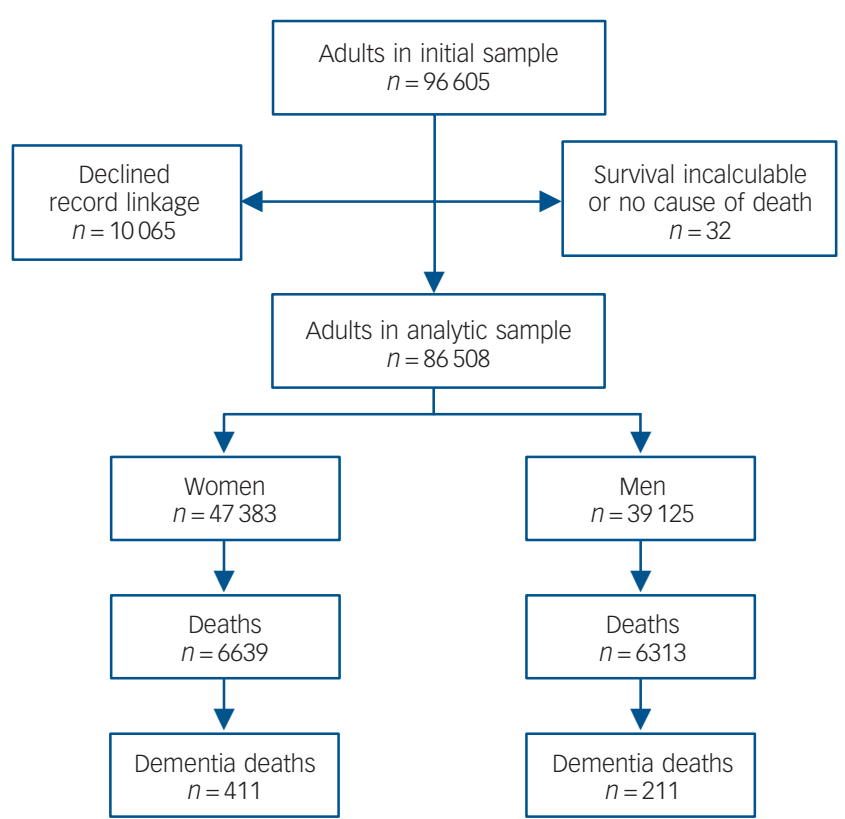

Fig. 1 Flow chart of participants from initial pooled sample through to analytic sample showing subsequent mortality: the Health Survey for England 1994-2004.

psychological distress. There was little association between occupational social class and alcohol consumption. Individuals from a lower occupational social class were more likely, as anticipated, to have spent less time in full-time education (women $r=0.41, P<0.001$; men $r=0.43, P<0.001)$. Similar patterns of association were seen with education as the exposure of interest (results not shown but available from the authors on request).

Of the 12952 deaths during a mean follow-up of 8.6 years (s.d.=3.5), 622 were ascribed to dementia. Figures 2 and 3 show the fully adjusted hazard ratios for the association of occupational social class and educational attainment respectively, with dementia death for each cohort study, in addition to gender- specific totals and meta-analysed effects. Overall, relative to study members from professional/intermediate occupational social classes, there was no increase in the risk of dementia death among those belonging to the lower social classes in women (fully adjusted hazard ratio (HR) for skilled non-manual: $\mathrm{HR}=0.88$ (95\% CI 0.59-1.31); skilled manual: $\mathrm{HR}=0.61(95 \%$ CI $0.36-1.06)$; semiskilled and unskilled manual: $\mathrm{HR}=0.92(95 \% \mathrm{CI} 0.62-1.36))$ or men (skilled non-manual: $\mathrm{HR}=1.03$ (95\% CI 0.53-2.00); skilled manual: $\mathrm{HR}=1.03$ (95\% CI 0.63-1.69); semi-skilled/unskilled manual: $\mathrm{HR}=1.33$ (95\% CI $0.80-2.21)$, Fig. 2). Relative to study members who left school aged 16 or older, there was an increase in the risk of dementia death among those leaving school earlier in women (leaving school aged 15: HR $=1.64$ (95\% CI 1.02-2.65); leaving school aged 14 or younger: $\mathrm{HR}=1.76(95 \%$ CI $1.23-$ 2.53)) but not in men (leaving school aged 15: $\mathrm{HR}=0.98(95 \%$ CI $0.51-1.88)$; leaving school aged 14 or younger: $\mathrm{HR}=1.20$ (95\% CI 0.77-1.87), Fig. 3).

Table 3 shows the impact of controlling for covariates on the association between the two indicators of socioeconomic status and dementia in women and men. The association between occupational social class and dementia death seen in age-adjusted models was completely explained by covariates in women but was more robust to statistical adjustment in men. The association between leaving full-time education at an earlier age and later dementia death observed in the age-adjusted models was fully 


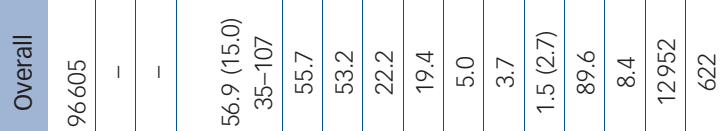

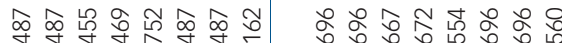

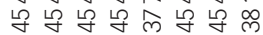

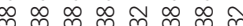

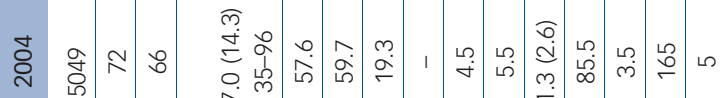

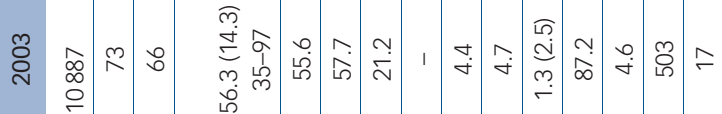

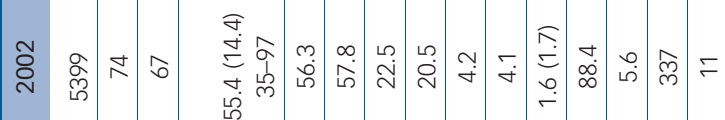

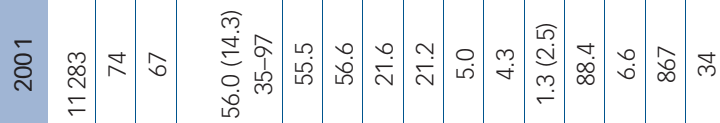

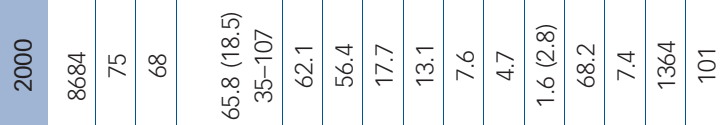

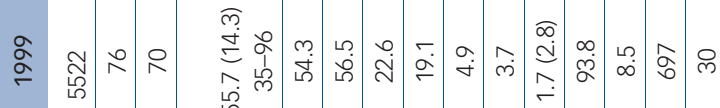

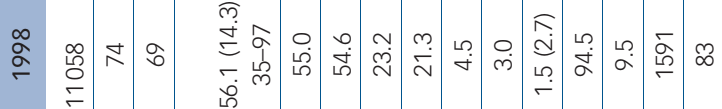

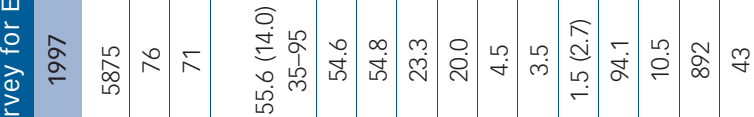

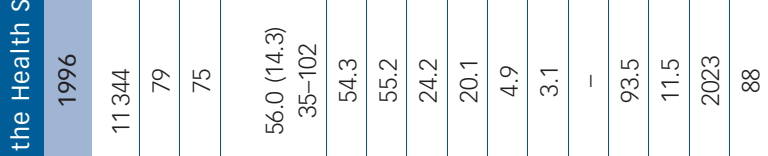

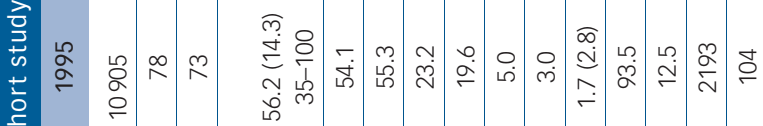

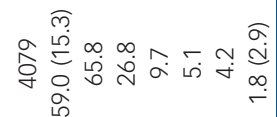

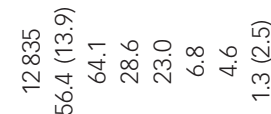

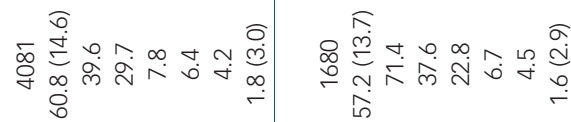

$\geq$

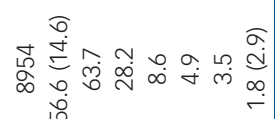

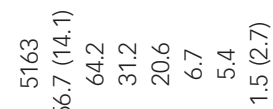

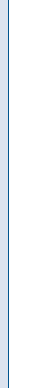

离

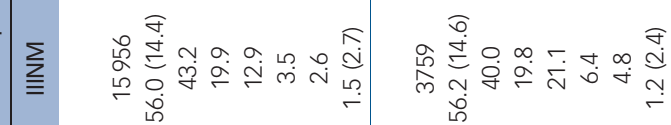

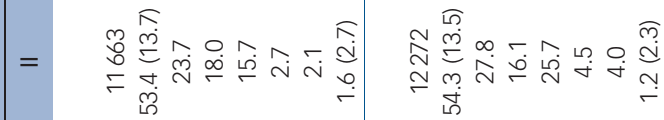

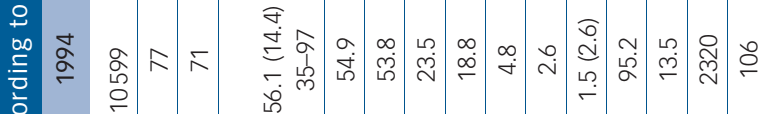

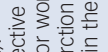

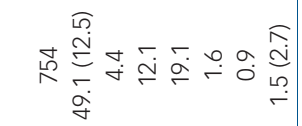

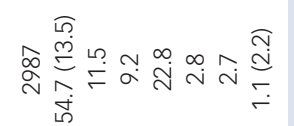
安

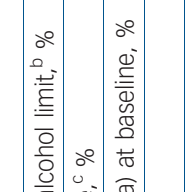


Study Participants $\begin{array}{cc}\text { Dementia } & \text { (a) } \\ \text { deaths } & \text { HR }\end{array}$

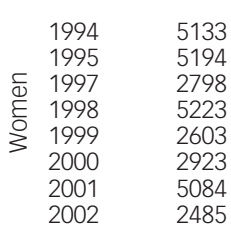

Summary 31343

$$
\begin{array}{r}
61 \\
58 \\
30 \\
37 \\
18 \\
38 \\
17 \\
5
\end{array}
$$

264
$0.88(0.59-1.31)$

Dementia

$\begin{array}{cr}\text { Study } & \text { Participants } \\ 1994 & 4407 \\ 1995 & 4602 \\ 1997 & 2393 \\ \overline{\frac{D}{d}} 1998 & 4447 \\ 1999 & 2254 \\ 2000 & 2383 \\ 2001 & 4313 \\ 2002 & 1991\end{array}$

Summary 26790

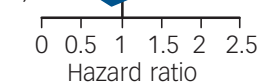

$\stackrel{\text { (a) }}{\mathrm{HR}}(95 \% \mathrm{Cl})$

$0.61(0.3-1.39)$
$1.57(0.7-3.46)$

$0.96(0.3-3.43)$

$0.81(0.3-2.27)$

$0.76(0.2-2.97)$

$0.57(0.2-1.38)$

$4.15(0.4-8.57)$

$$
\text { Hazard ratio }
$$

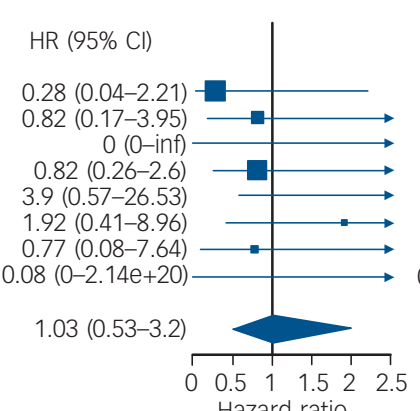

$2.21 \mathrm{e}+8(0.0-\mathrm{in})$

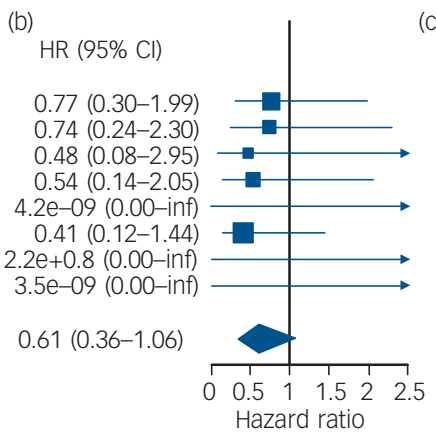

(C)
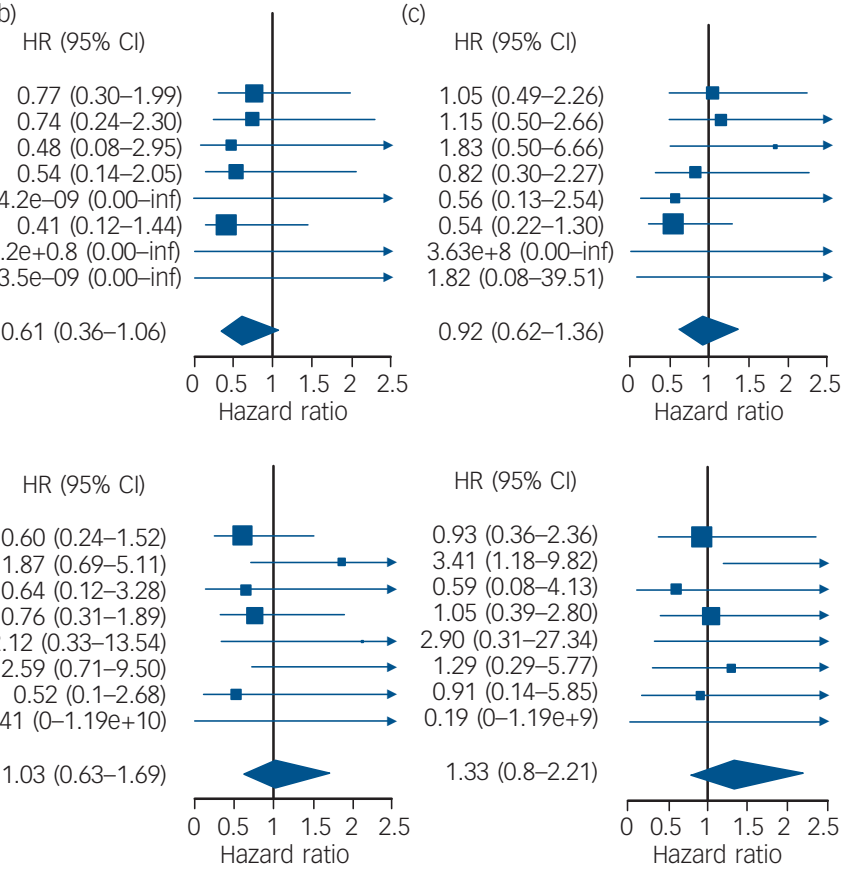

Fig. 2 Fully adjusted hazard ratios (HRs) with 95\% confidence intervals of dementia death by survey year for individuals, from left to right, from (a) skilled non-manual, (b) skilled manual and (c) semi-skilled/unskilled manual occupational social classes compared with professional/intermediate: the Health Survey for England 1994-2004.

For women: $I^{2}$ values for these models are $4.1 \%, 0.0 \%$ and $0.0 \%$. For men: $I^{2}$ values for these models are $0.0 \%, 12.9 \%$ and $6.4 \%$.

\begin{tabular}{|c|c|c|}
\hline \multirow{10}{*}{$\stackrel{\frac{\digamma}{\mathcal{Q}}}{\Sigma}$} & Study $\quad P$ & Participants \\
\hline & 1994 & 4407 \\
\hline & 1995 & 4602 \\
\hline & 1997 & 2393 \\
\hline & 1998 & 4447 \\
\hline & 1999 & 2254 \\
\hline & 2000 & 2383 \\
\hline & 2001 & 4313 \\
\hline & 2002 & 1991 \\
\hline & Summary & 26790 \\
\hline
\end{tabular}

$\begin{array}{cccc}\text { Study } & \text { Participants } & \begin{array}{c}\text { Dementia } \\ \text { deaths }\end{array} \\ & & 5133 & 61 \\ & 1994 & 5194 & 58 \\ \bar{\Phi} & 1995 & 2798 & 30 \\ \overline{\overline{0}} & 1997 & 5223 & 37 \\ \overline{3} & 2603 & 18 \\ & 1999 & 2823 & 38 \\ & 2000 & 5084 & 17 \\ 2001 & 2485 & 5 \\ & & \\ & & & \end{array}$

33
34
11
36
11
20
9
1
155

(a) $\mathrm{HR}(95 \% \mathrm{Cl})$

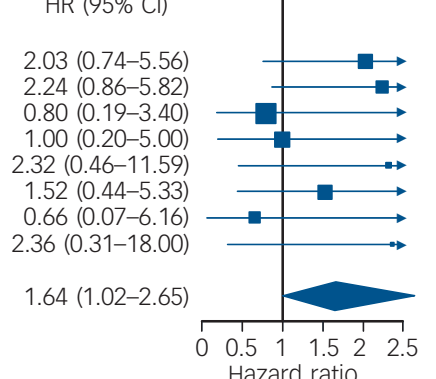

Hazard ratio

Dementia
deaths $\quad H R(95 \% ~ C l)$

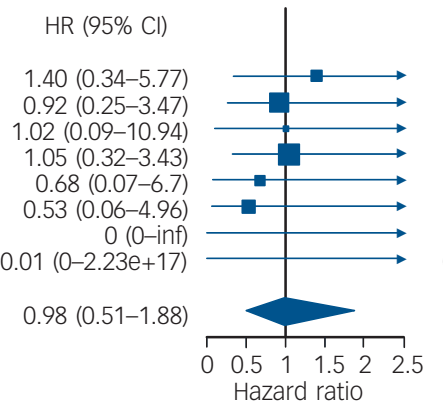

(b)

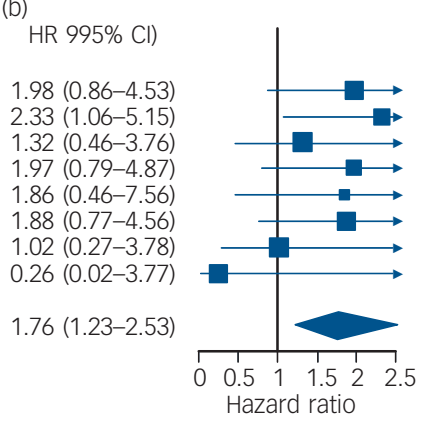

$\mathrm{HR}(95 \% \mathrm{Cl})$

$1.53(0.60-3.90)$ $1.02(0.37-2.83)$ $1.17(0.21-6.41)$ $0.93(0.41-2.12)$ $0.60(0.12-2.97)$

$1.60(0.47-5.49)$ $7.80(0.80-75.98)$ $0.00(0-1.69 \mathrm{e}+28)$

$1.20(0.77-1.87)$

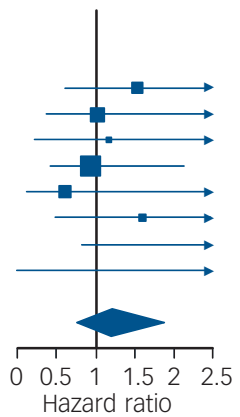

Fig. 3 Fully adjusted hazard ratios (HRS) with 95\% confidence intervals of dementia death by survey year for individuals who left full-time education aged, from left to right, (a) 15 years and (b) 14 years or younger compared with those who left full-time education aged 16 years or older: the Health Survey for England 1994-2004.

For women: $I^{2}$ values for these models are $0.0 \%$ and $0.0 \%$. For men: $I^{2}$ values for these models are $0.0 \%$ and $0.0 \%$.

attenuated on adjustment for covariates in men but remained in women.

\section{Sensitivity and subgroup analyses}

Data were missing for one or more variables in 32.8\% $(n=28375)$ of the sample. Online Table DS1 shows a comparison of the characteristics of individuals with complete data for all variables $v$. individuals who were missing data for one or more variables. Individuals with complete data were more likely to be male, younger, drink more alcohol per week and were more likely to drink over the recommended limits and a larger proportion were current smokers. They were less likely to have diabetes and 


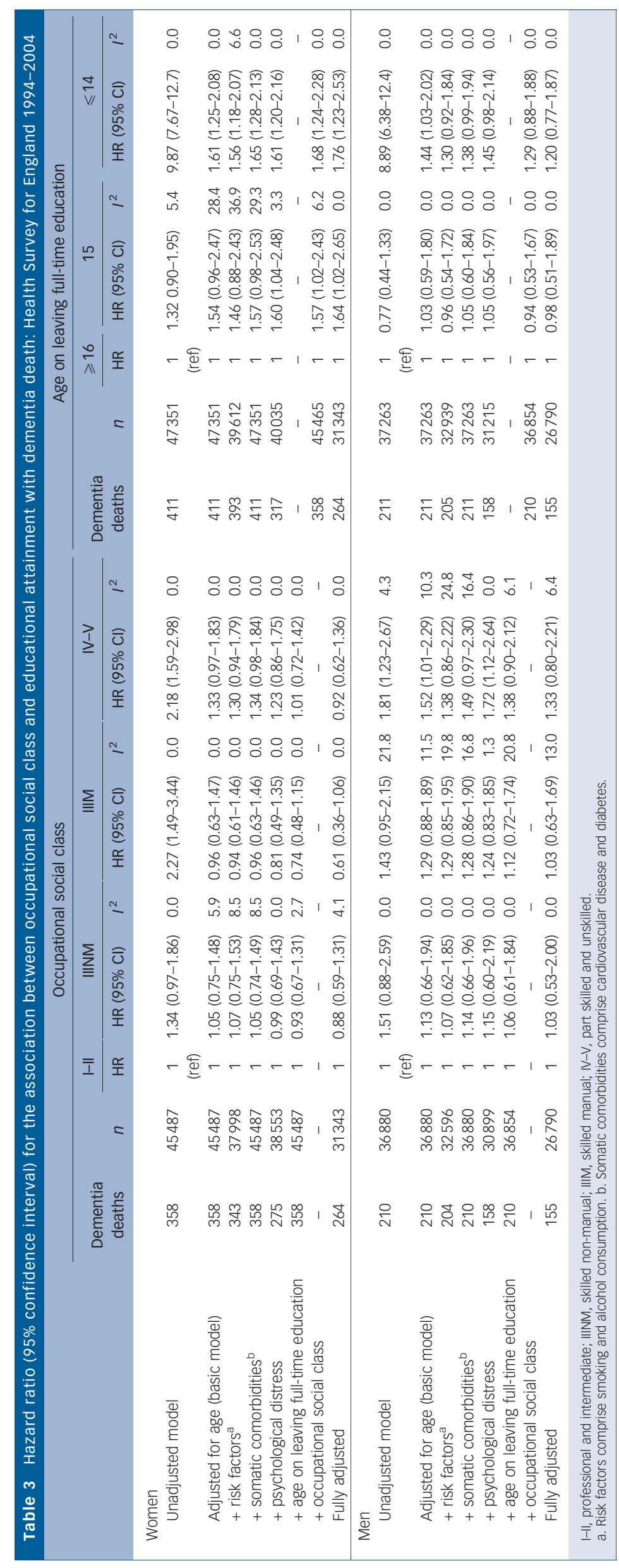

cardiovascular disease at baseline. A slightly larger proportion of individuals with complete data belonged to a non-manual occupational social class and women were less likely to have left school early (the reverse was true of men). Therefore, individuals with missing data did not always have unfavourable levels of risk factors. Accounting for missing data by multiple imputation did not alter our conclusions (online Table DS2). Similarly, excluding dementia-related deaths occurring within 5 years of follow-up, to explore reverse causality in the case of current occupational social class, did not affect our results (online Table DS3).

\section{Discussion}

The purposes of the current study were to show that an individual participant meta-analysis technique can be applied to a socioeconomic risk factor and also to investigate the association between socioeconomic status and dementia-related death. In order to achieve the former we used a convenience sample of 11 large UK population-based cohort studies. With regard to the latter, the main finding of this study was that educational attainment was associated with dementia death in women but not in men. In men, we also found that, relative to the highest two classes, belonging to the lowest two occupational social classes was associated with an elevated risk of dementia death; a relationship that did not reach statistical significance at conventional levels. There was no association between occupational social class and dementia death in women.

\section{Strengths and limitations}

To our knowledge, this study is the first to use an individual participant meta-analysis methodology to examine the association between socioeconomic variables and dementia. In contrast to previous literature-based analyses, an individual participant meta-analysis has the advantage of providing precise estimates of risk marker-disease relationships, reliable information on the shape of a given risk factor-disease relationship (for example dose-response $v$. threshold) and a consistent approach to statistical control for plausible covariates and subgroup analyses. We used a large, well-characterised sample that is representative of the general population in England. This provided sufficient power to allow gender-specific analyses and allowed us to explore the role of a series of explanatory factors. By including two measures of socioeconomic status at different time points we offer some insights into the influence of life-course socioeconomic status on dementia risk.

This notwithstanding, the data have their limitations. Despite the large sample and over 12000 deaths during follow-up, there were only 622 dementia-related deaths. This is due to the wide age range of individuals included in the survey at baseline - adults aged 35 and over. Although this wide age range increases the generalisability of the findings, it has resulted in a relatively small proportion of individuals dying with dementia and consequently wide confidence intervals, limiting the conclusions that can be drawn. The identified gender differentials should be viewed in the light of this question of power. The number of dementiarelated deaths in this study is likely to have been additionally affected by the unavoidable problems of underdiagnosis of dementia in the community, ${ }^{23}$ underrecording of dementia on death certificates ${ }^{24}$ and diagnoses being inaccurately coded. Non-recording of dementia on death certificates has been highlighted as an important issue, but this seems to be improving. A recent study identified that $71.5 \%$ of a clinic sample diagnosed with probable Alzheimer's disease had dementia correctly 
recorded on their death certificate. ${ }^{25}$ There was no association in that sample between correct dementia certification and area deprivation or premorbid IQ estimated by the National Adult Reading Test (unpublished results available from the author on request), suggesting that individuals reported as having dementia on their death certificate are representative of the population of people with diagnosed dementia in the community, at least in terms of intelligence and level of deprivation.

\section{Comparison with previous studies}

Dementia was estimated to affect over 24 million people globally in 2001 and this is expected to rise to over 80 million by $2040 .{ }^{26}$ In England and Wales the prevalence of dementia is estimated to vary from approximately $1.5 \%$ at age $65-69$ to approximately $25.3 \%$ over the age of $84 .{ }^{27}$ Dementia incidence ranges from $7.4(95 \%$ CI 3.6-16.1) per 1000 person-years at age 65-69 to 84.9 (95\% CI $63.0-107.8)$ per 1000 person-years over the age of $84 .^{28}$ However, existing prospective studies of socioeconomic factors have generally been small in scale - only one analysis reported a combined sample larger than $10000^{19}$ - and few studies measure socioeconomic status at more than one time point. ${ }^{8}$ On a related note, the association between education and dementia risk has been reported to be present in women but not in men; ${ }^{19}$ it is therefore important that studies are sufficiently large to allow gender-specific analyses. In addition, for older participants - who are most likely to have died with dementia during follow-up - the social class may have been allocated to females on the basis of their husband's occupation, further muddying the waters.

Factors across the lifespan have been implicated in the aetiology of dementia. A series of studies confirm a link between early life socioeconomic status and dementia risk with early parental death - and, potentially, consequent socioeconomic hardship - being highlighted as an important risk factor. ${ }^{29}$

A total of 51 of 88 studies included in a systematic review ${ }^{6}$ reported a significant association between a basic education and dementia risk, the remainder reporting no effect. However, only two previous studies report gender-specific effects, showing an effect of education on dementia risk in women but not in men, as found in the current study.

In England, the Education Act 1918 raised the school leaving age from 12 to 14 . It was further increased to 15 in 1947 and to 16 in 1973. Participants in the Health Survey for England came from all these educational eras and overall approximately half of men and women stayed on after completion of the compulsory period of education. However, individuals were much more likely to remain in education longer if they were born later: $86.7 \%$ of women and $87.2 \%$ of men born after 1956 (who therefore had to remain in school until 16) compared with $17.8 \%$ of women and $18.5 \%$ of men born before 1906 and who could therefore leave school at 12 . However, the reference category used, of individuals who remained in full-time education up to 16 years or later, exactly matches those who remained in education after the compulsory school leaving age.

Since children scoring higher on IQ tests, as well as children from a higher occupational social class, are more likely to be given the opportunity to remain in education for longer, a number of studies have investigated the association between childhood mental ability and dementia. A Scottish study identified 50 participants in the 1932 Scottish Mental Survey who had developed dementia and ascertained that their scores on the Moray House Test in 1932 had been significantly lower than their peers in the same area who did not develop dementia. ${ }^{30}$ However, a larger subsequent study confirmed that this was the case for vascular dementia but not for Alzheimer's disease. ${ }^{31}$ On the other hand, the Nun study identified that low linguistic ability at a mean age of 22 was associated with Alzheimer's disease in the 14 study participants aged 79-96 whose brains were neuropathologically examined post-mortem. ${ }^{32}$

Occupation in adulthood has also been shown to be associated with dementia risk - high 'occupational attainment' is associated with lower dementia risk. ${ }^{33}$ However, since the pathology of Alzheimer's disease and probably vascular dementia develops over a very long period of time ${ }^{34}$ risk factors must be measured sufficiently early in life for them to have an effect, and exposures immediately before retirement may have little or no effect on dementia risk.

Few studies measure socioeconomic status across the whole lifespan. One study to do so identified early parental death, manual work and physical illness in the spouse or serious illness in a child, both after the age of 65 , to be independent risk factors for dementia. ${ }^{8}$ However, many of the socioeconomic factors measured in studies are closely linked and disentangling their individual effects can be extremely difficult.

\section{Mechanisms of effect}

The role of education and mental ability as potential risk factors for dementia has been linked to the hypothesis of cognitive reserve - that certain individuals' brains are structurally or functionally more resilient to disease or injury. This sprang from the observation that there was no clear relationship between the extent of brain pathology and the clinical manifestations of dementia in an individual. Cognitive reserve might relate to a person's intrinsic make up or could result from external experience, i.e. education and occupation. Indeed, there is evidence that occupation is associated with differences in parietal blood flow in Alzheimer's disease that could be a marker of reserve. $^{35}$

Criticisms of the cognitive reserve hypothesis include the suggestion that the effect merely reflects performance on cognitive tests $^{36}$ or that the effect is mediated by lifestyle factors and cerebrovascular risk. ${ }^{37}$ The present study used clinical diagnoses of dementia recorded on death certificates and two measures of socioeconomic status at different stages of life. This allows us to demonstrate that the observed association between education and dementia in women was not mediated by adult occupation.

Since an individual with more cognitive reserve would have more advanced pathological changes at the time of diagnosis, it has been suggested that this could be linked with a swifter decline and poorer survival. Some studies have found that individuals with Alzheimer's disease and higher educational and occupational attainment declined faster in their performance on cognitive tests. ${ }^{38}$ This aspect of the cognitive reserve hypothesis was not examined by the current study.

\section{Implications}

An association between lower educational attainment and dementia in women but not men has a number of implications. First, the mechanism of this association, currently hypothesised to relate to cognitive reserve, must be clarified. Second, the reason that the association is observed only in women should be investigated. It may be that fewer women entered further education and that those who did had to be very intelligent to do so. Thus, length of education in these cohorts could be confounded by intelligence in women to a greater extent than men. However, the identified gender difference may also potentially hold the clue to an intervention to decrease the risk 
of dementia in women, if a modifiable risk (or protective) factor could be identified. Lastly, if this association were to reflect a causative link between education and dementia risk, promoting higher and further education, especially for women, could have important public health consequences for a common and serious condition.

In conclusion, this large prospective study shows an association between leaving full-time education at a younger age and dementia death in women, but not in men. This relationship remained after adjustment for alcohol, smoking, cardiovascular disease, diabetes, psychological distress and occupational social class. An association between lower occupational social class and dementia death that did not reach statistical significance at conventional levels was also observed in men, but not in women.

Tom C. Russ, MRCPsych, Alzheimer Scotland Dementia Research Centre, University of Edinburgh; Scottish Dementia Clinical Research Network, NHS Scotland; and Centre for Cognitive Ageing \& Cognitive Epidemiology, University of Edinburgh; Emmanuel Stamatakis, PhD, Mark Hamer, PhD, Department of Epidemiology and Public Health, University College London; John M. Starr. FRCPEd, Alzheimer Scotland Dementia Research Centre, University of Edinburgh; Scottish Dementia Clinical Research Network, NHS Scotland; and Centre for Cognitive Ageing \& Cognitive Epidemiology, University of Edinburgh; Mika Kivimäki, PhD, Department of Epidemiology and Public Health, University College London; G. David Batty, PhD, Centre for Cognitive Ageing \& Cognitive Epidemiology, University of Edinburgh; and Department of Epidemiology and Public Health, University College London, UK

Correspondence: Tom C. Russ, Alzheimer Scotland Dementia Research Centre University of Edinburgh, 7 George Square, Edinburgh EH8 9JZ, UK. Email: tom.russ@nhs.net

First received 14 Aug 2012, final revision 18 Feb 2013, accepted 19 Feb 2013

\section{Funding}

This work was supported by Alzheimer Scotland. T.C.R. is supported by Alzheimer Scotland and is employed in the National Health Service by the Scottish Dementia Clinical Research Network, which is funded by the Chief Scientist Office (part of the Scottish Government Health Directorates). T.C.R. and J.M.S. are members of the Alzheimer Scotland Dementi Research Centre funded by Alzheimer Scotland. T.C.R., J.M.S. and G.D.B. are members of the University of Edinburgh Centre for Cognitive Ageing and Cognitive Epidemiology, part of the cross-council Lifelong Health and Wellbeing Initiative (G0700704/84698). Funding from the Biotechnology and Biological Sciences Research Council, Engineering and Physical Sciences Research Council, Economic and Social Research Council and Medical Research Council is gratefully acknowledged. E.S. is supported by a National Institute for Health Research Career Development Fellowship. M.K. is supported by the Medical Research Council, UK, the US National Institutes of Health (R01HL036310; R01AG034454), the Academy of Finland, Finland, and by a professorial fellowship from the Economic and Social Research Council, UK. G.D.B. was a Wellcome Trust Fellow during the preparation of this manuscript.

\section{References}

1 Marmot MG, Bosma $\mathrm{H}$, Hemingway $\mathrm{H}$, Brunner $\mathrm{E}$, Stansfeld $\mathrm{S}$. Contribution of job control and other risk factors to social variations in coronary heart disease incidence. Lancet 1997; 350: 235-9.

2 Stringhini S, Dugravot A, Kivimaki M, Shipley M, Zins M, Goldberg M, et al. Do different measures of early life socioeconomic circumstances predict adult mortality? Evidence from the British Whitehall II and French GAZEL studies. J Epidemiol Community Health 2011; 65: 1097-103.

3 Lorant $\mathrm{V}$, Deliege $\mathrm{D}$, Eaton $\mathrm{W}$, Robert $\mathrm{A}$, Philippot $\mathrm{P}$, Ansseau $\mathrm{M}$. Socioeconomic inequalities in depression: a meta-analysis. Am J Epidemiol 2003; 157: 98-112.

4 Fryers $T$, Melzer D, Jenkins R. Social inequalities and the common mental disorders. Soc Psychiatry Psychiatr Epidemiol 2003; 38: 229-37.

5 Jokela M, Batty GD, Vahtera J, Elovainio M, Kivimäki M. Socioeconomic inequalities in common mental disorders and psychotherapy treatment in the UK between 1991 and 2009. Br J Psychiatry 2013; 202: 115-20.

6 Sharp ES, Gatz M. Relationship between education and dementia: an updated systematic review. Alzheimer Dis Assoc Disord 2011; 25: 289-304.

7 Qiu CX, Karp A, von Strauss E, Winblad B, Fratiglioni L, Bellander T. Lifetime principal occupation and risk of Alzheimer's disease in the Kungsholmen Project. Am J Ind Med 2003; 43: 204-11.

8 Persson G, Skoog I. A prospective population study of psychosocial risk factors for late onset dementia. Int J Geriatr Psychiatry 1996; 11: 15-22.
9 Karp A, Kåreholt I, Qiu C, Bellander T, Winblad B, Fratiglioni L. Relation of education and occupation-based socioeconomic status to incident Alzheimer's disease. Am J Epidemiol 2004; 159: 175-83.

10 Mindell J, Biddulph JP, Hirani V, Stamatakis E, Craig R, Nunn S, et al. Cohort profile: the Health Survey for England. Int J Epidemiol 2012; 41: 1585-93.

11 Macintyre S, McKay L, Der G, Hiscock R. Socio-economic position and health: what you observe depends on how you measure it. J Public Health 2003; 25: 288.

12 Royal College of Physicians of London. A Great and Growing Evil: The Medical Consequences of Alcohol Abuse. Royal College of Physicians of London, 1987.

13 Goldberg DP, Gater R, Sartorius N, Ustun T, Piccinelli M, Gureje O, et al. The validity of two versions of the GHQ in the WHO study of mental illness in general health care. Psychol Med 1997; 27: 191-7.

14 Aalto A-M, Elovainio M, Kivimaki M, Uutela A, Pirkola S. The Beck Depression Inventory and General Health Questionnaire as measures of depression in the general population: a validation study with the Composite International Diagnostic Interview as the gold standard. Psychiatry Res 2012; 197: 163-71.

15 Russ TC, Hamer M, Stamatakis E, Starr JM, Batty GD. Psychological distress as a risk factor for dementia death. Arch Intern Med 2011; 171: 1858-9.

16 World Health Organization. Manual of the International Statistical Classification of Diseases, Injuries, and Causes of Death (9th revision). WHO, 1977.

17 World Health Organization. ICD-10: International Statistical Classification of Diseases and Related Health Problems (10th revision). WHO, 1992.

18 Cox DR. Regression models and life-tables. J Roy Stat Soc B 1972; 34 187-220.

19 Launer L, Andersen K, Dewey ME, Letenneur L, Ott A, Amaducci L, et al. Rates and risk factors for dementia and Alzheimer's disease. Neurology 1999; 52: 78-84.

20 Batty GD, Der G, Macintyre S, Deary IJ. Does IQ explain socioeconomic inequalities in health? Evidence from a population based cohort study in the west of Scotland. BMJ 2006; 332: 580-4.

21 Viechtbauer W. Conducting meta-analyses in $\mathrm{R}$ with the metafor package. Stat Softw 2010; 36: 1-48.

22 von Elm E, Altman D, Egger M, Pocock SJ, Gøtzsche PC, Vandenbroucke JP. Strengthening the reporting of observational studies in epidemiology (STROBE) statement: guidelines for reporting observational studies. BMJ 2007; 335: 806-8.

23 Sampson EL, Blanchard MR, Jones L, Tookman A, King M. Dementia in the acute hospital: prospective cohort study of prevalence and mortality. $\mathrm{Br} J$ Psychiatry 2009; 195: 61-6.

24 Martyn CN, Pippard EC. Usefulness of mortality data in determining the geography and time trends of dementia. J Epidemiol Community Health 1988; 42: 134.

25 Russ TC, Batty GD, Starr JM. Cognitive and behavioural predictors of survival in Alzheimer disease: results from a sample of treated patients in a tertiaryreferral memory clinic. Int J Geriatr Psychiatry 2012; 27: 844-53.

26 Ferri $\mathrm{CP}$, Prince $\mathrm{M}$, Brayne $\mathrm{C}$, Brodaty $\mathrm{H}$, Fratiglioni L, Ganguli M, et al. Global prevalence of dementia: a Delphi consensus study. Lancet 2006; 366 2112-7

27 MRC CFAS. Cognitive function and dementia in six areas of England and Wales: the distribution of MMSE and prevalence of GMS organicity level in the MRC CFA Study. Psychol Med 1998; 28: 319-35.

28 Matthews F, Brayne C. The incidence of dementia in England and Wales: findings from the five identical sites of the MRC CFA Study. PLOS Med 2005; 2: e193.

29 Norton MC, Ostbye T, Smith KR, Munger RG, Tschanz JT. Early parental death and late-life dementia risk: findings from the Cache County Study. Age Ageing 2009; 38: 340-3.

30 Whalley $\mathrm{L}$, Starr JM, Athawes R, Hunter D, Pattie A, Deary IJ. Childhood mental ability and dementia. Neurology 2000; 55: 1455-9.

31 McGurn B, Deary IJ, Starr JM. Childhood cognitive ability and risk of lateonset Alzheimer and vascular dementia. Neurology 2008; 71: 1051-6.

32 Snowdon DA, Kemper SJ, Mortimer JA, Greiner LH, Wekstein DR, Markesbery WR. Linguistic ability in early life and cognitive function and Alzheimer's disease in late life - findings from the Nun Study. JAMA 1996; 275: 528-32.

33 Stern Y, Gurland B, Tatemichi TK, Tang MX, Wilder D, Mayeux R. Influence of education and occupation on the incidence of Alzheimer's Disease. JAMA 1994: 271: 1004-10.

34 Braak H, Braak E. Frequency of stages of Alzheimer-related lesions in different age categories. Neurobiol Aging 1997; 18: 351-7. 
35 Stern $\mathrm{Y}$, Alexander G, Prohovnik I, Stricks L, Link B, Lennon M, et al. Relationship between lifetime occupation and parietal flow. Neurology 1995 45: 55-60.

36 Gilleard C. Education and Alzheimer's disease: a review of recent international epidemiological studies. Aging Ment Health 1997; 1: 33-46.
37 Del Ser T, Hachinski V, Merskey H, Munoz DG. An autopsy-verified study of the effect of education on degenerative dementia. Brain 1999; 122: 2309-19.

38 Stern $Y$, Albert S, Tang MX, Tsai WY. Rate of memory decline in AD is related to education and occupation: cognitive reserve? Neurology 1999; 53 1942-7.

\section{poems \\ by doctors}

\section{Endless Night}

\section{Shaun Love}

These winter nights lie long and cold before you, like an icy road

that grants your well worn wheels no hold. And so you spin

around and round, tossed to and fro, in frozen sin.

You rev your engine, not to gain some hoped for ground, instead, to drain your heart of salted slushy pain.

But it won't bate.

For what goes round, comes back in veins and circulates.

Your heart alone can not reduce your load of pain. You need to use some organs that change old for new. Light, warm, fresh air

is waiting where you travel to on this black glacier.

An astronaut sent through such space, would, with drugs, dilute to trace the time and mind the travel takes. But drugs won't work. You'd fall asleep, only to wake back at the start.

Others sleep without a sound.

You hear their silence all around, sliding over the frictionless ground. Blind to night's stare,

deaf to their own baying hounds. Life is not fair.

So curse the Gods that made you be but gave you eyes so you could see, the distance that exists between the man you are and the man they made your eyes to see as mankind's par.

But pull and turn this rage inside to burn the bane of your poor mind. Your ice will melt. Your time will bide. The sun goes down.

Another summer sinks behind the long drawn blackout blind.

c) Shaun Love 